\title{
Autoconcepto y conducta antisocial en adolescentes del programa de prevención del delito del Ministerio Público de Lima, 2016
}

\author{
Sayda Noemi Arosquipa Soncco ${ }^{1}$ \\ Universidad Peruana Unión ${ }^{1}$
}

Recibido: 18 de marzo de 2016

Aceptado: 18 de julio de 2016

\begin{abstract}
Resumen
El objetivo del presente estudio fue determinar la relación entre el autoconcepto y la conducta antisocial en adolescentes del programa prevención del delito del Ministerio Público de Lima. Se empleó el muestreo no probabilístico y la muestra estuvo conformada por 125 adolescentes, varones y mujeres cuyas edades oscilan entre 14 a 19 años. El diseño es no experimental, de corte transversal y alcance correlacional. Se utilizó el cuestionario de Autoconcepto (AF-5) elaborado por García y Musitu (2014) y el Cuestionario de conducta antisocial - delictiva (A-D) de Seisdedos (2001). Los resultados obtenidos indican que existe relación altamente significativa e inversa (rho= ,$- 249^{* *}$ y $p<0,005$ ) entre autoconcepto y conducta antisocial; esto quiere decir que a menor autoconcepto mayor presencia de conductas antisociales. Del mismo modo, se halló relación significativa entre las dimensiones de autoconcepto académico y emocional con la conducta antisocial. Por otro lado, se observa que no hay relación entre las dimensiones física, familiar y social con la conducta antisocial. Finalmente, se concluye que existe relación entre autoconcepto y conducta antisocial.
\end{abstract}

Palabras Claves: Conducta antisocial, autoconcepto, delito, adolescencia

\section{Self-concept and antisocial behavior in adolescents of the crime prevention program of the Public Ministry of Lima, 2016}

\begin{abstract}
\footnotetext{
${ }^{1}$ Correspondencia al autor

E-mail: sayda.arosquipa@gmail.com
}

The objective of the present study is to determine the relationship between self-concept and antisocial behavior in adolescents of the crime prevention program of the Public 
Ministry of Lima. Non-probabilistic sampling was used and the sample consisted of 125 adolescents, males and females whose ages ranged from 14 to 19 years of age. The design is non-experimental, with a cross-sectional and correlational scope. The selfconcept questionnaire (AF-5) elaborated by Garcia and Musitu (2014) and the AntiSocial-Criminal Conduct Questionnaire (A-D) by Seisdedos (2001) were used. The results indicate that there is a highly significant and inverse relationship (rho $=-.249 * *$ and $\mathrm{p}<0.005$ ) between self-concept and antisocial behavior. This means that lower selfconcept levels correspond to an increased presence of antisocial behaviors. Furthermore, the significant relationship between the dimensions of academic and emotional self-concept with antisocial behavior was found. On the other hand, it is observed that there is no relationship in the physical, family and social dimensions. In conclusion, there is a relationship between self-concept and antisocial behavior.

Keywords: Antisocial behavior, self-concept, adolescence

\section{Introducción}

En la actualidad las conductas de riesgo en adolescentes son de amplia preocupación para la población y los profesionales que estudian dicha problemática. Frente a lo descrito, Esnaola, Goñi y Madariaga (2008) señalan que los adolescentes experimentan cambios psicosociales y que la formacion del autoconcepto juega un papel trascendental en la búsqueda de la identidad que reafirma la personalidad, permite el bienestar psicológico y el ajuste social. De modo que, si los adolescentes tienen un autoconcepto negativo se podría presentar en ellos un conjunto de manifestaciones o conductas desadaptativas o antisociales (Krug, Dahlberg, Mercy, Zwi y Lozano, 2003). Asimismo, la teoria de Reckless (1961) sostiene que un autoconcepto debil o negativo lo situa en una posicion de vulnerabilidad frente a factores prodelictivos. La conducta antisocial es definido como un conjunto diverso de acciones inadecuades tales como agresiones verbales y fisicas, mentiras, hurto, vandalismo y cualquier conducta que infrinja las reglas y normas sociaes contra cualquier individuo o su entorno (Kazdin y Buela-Casal, 2002).

Según la OMS, las estadísticas a nivel mundial mostraron que en el año 2000 se produjo un total de 199000 homicidios de jóvenes, es decir que 565 adolescentes y adultos jóvenes entre edades de 19 a 29 años murieron cada día resultado de la violencia interpersonal. La violencia mortal ha ido en aumento y se produce por medio del uso frecuente de armas de fuego, así como la violencia no mortal que se produce por medio 
del uso de los puños, y otras armas como las punzo cortantes y los garrotes. En cuanto a la realidad Peruana Herrera y Morales (2005) señalan la existencia aproximadamente 12,795 pandilleros entre 12 y 24 años de edad, agrupados en 390 pandillas juveniles. Sin embargo, estas cifras han ido en aumento hasta la actualidad, según el Ministerio del Interior (2013) menciona que la delincuencia ha aumentado en un $20 \%$.

El autoconcepto y la conducta antisocial han sido estudiadas generalmente por separado con otras variables similares, tales como los problemas de conducta y ajuste social, se puede citar por ejemplo al estudio de Garaigordobil, Dura y Pérez (2005) en el que relacionan los sintomas psicopatológicos, problemas de conducta con autoconcepto y autoestima en adolescentes. Los resultados sugieren que los adolescentes con alto autoconcepto y alta autoestima tienen baja presencia de síntomas psicopatológicos y de problemas de conducta. Asimismo, Fuentes, García y Lila (2011) estudiaron el autoconcepto y ajuste psicosocial en la adolescencia, donde los resultados indicaron que a mayor autoconcepto mejor ajuste psicológico, buena competencia personal y menos problemas comportamentales. Por ultimo Carrion, E. (2015) desarrolló la investigacion de conductas antisociales y clima social escolar en estudiantes del distrito de Florencia de Mora en Perú. Los resultados muestran que existe una correlación positiva débil no significativa, entre las variables conductas antisociales y clima social escolar.

Los estudios de relación entre del autoconcepto y la conducta antisocial son escasos. Sin embargo, una investigacion realizada por Garcia e Inmaculada (2016) desarrolló las dos variables en alumnos de secundaria. Los resultados mostraron que sí existe relación significativa. Se infiere que debido a la complejidad de la delimitancion del concepto de conducta antisocial se imposibilita la formación de investigaciones, como lo refiere Kazdin y Buela-Casal (2002). De la misma manera, no se encontraron antecedentes que hayan estudiado una población en riesgo, puesto que la mayoria solo se centra en el ámbito academico o escolar. (Alafaro, 2009; Portocarrero, 2014; García y Inmaculada, 2016 y Fuentes, Gracia y Lila, 2011)

Basado en la necesidad de estudios al respecto y a la importancia del asunto, el objetivo de esta investigacion fue determinar si existe relación significativa entre autoconcepto y conducta antisocial en adolescentes del programa de prevención del delito del Ministerio Público de Lima, 2016. Es así que la hipótesis general del estudio pretende determinar que sí existe relación 
estadísticamente significativa entre las dos variables. También se plantearon hipótesis específicas con cada una de las dimensiones del autoconcepto: académico, familiar, social, emocional y físico con la conducta antisocial. 


\section{Metodo}

\subsection{Participantes}

Para la selección de los participantes se utilizó el método no probabilístico por conveniencia. La muestra estuvo compuesta por 125 adolescentes de ambos sexos, cuyas edades oscila entre los 14 a 19 años.

En la Tabla 1, se observa que el $69,6 \%$ de la muestra son varones y 30,4\% son mujeres, con estudios de primaria y secundaria: avanzado, $1^{\circ} \mathrm{CEBA}, 2^{\circ} \mathrm{CEBA}$, $3^{\circ} \mathrm{CEBA}, 4^{\circ} \mathrm{CEBA}$ y $5^{\circ} \mathrm{EBR}$, pertenecientes a diferentes distritos de Lima Metropolitana: Los Olivos, Comas, San Juan de Lurigancho, Rímac, San Juan de Miraflores, Villa el Salvador, Villa María del Triunfo, La Victoria, Breña y Ate Vitarte.

Tabla 1

Análisis de frecuencia de las características de los participantes

\begin{tabular}{llll}
\hline Datos Sociodemográficos & & $\mathrm{f}$ & $\%$ \\
\hline Genero & Masculino & 87 & 69,6 \\
Grado académico & Femenino & 38 & 30,4 \\
& Avanzado & 6 & 4,8 \\
& $1^{\circ}$ CEBA & 18 & 15,2 \\
& $2^{\circ}$ CEBA & 45 & 36,0 \\
& $3^{\circ}$ CEBA & 36 & 28,8 \\
Edades & $4^{\circ}$ CEBA & 16 & 12,8 \\
& $5^{\circ}$ EBR & 3 & 2,4 \\
& 14 & 15 & 12,0 \\
& 15 & 22 & 17,6 \\
& 16 & 17 & 13,6 \\
& 17 & 25 & 20,0 \\
Zonas & 18 & 33 & 26,4 \\
& 19 & 13 & 10,4 \\
& Los Olivos & 20 & 16,0 \\
& Comas & 15 & 12,0 \\
& San Juan de Lurigancho & 18,4 \\
& Rímac & 10 & 8,0 \\
& San Juan de Miraflores & 16 & 12,8 \\
& Villa el Salvador & 14,4 \\
\hline
\end{tabular}




\begin{tabular}{llll}
\hline & Villa María del Triunfo & 8 & 6,4 \\
& La Victoria & 6 & 4,8 \\
& Breña & 5 & 4,0 \\
& Ate Vitarte & 4 & 3,2 \\
\hline
\end{tabular}

\subsection{Instrumentos}

Para la evaluación de la variable de Autoconcepto se utilizó la Escala de Autoconcepto forma 5 (AF5) cuyos autores son García y Musitu (2001) quienes aplicaron la prueba para medir la valides y confiabilidad. El instrumento se aplica de manera grupal o individual con una duración promedio de 15 minutos. Puede ser administrado en poblaciones de estudiantes de $5^{\circ}$ y $6^{\circ}$ grado del nivel primario, secundario, bachiller universitario y adultos no escolarizados. El instrumento está compuesto por 30 ítems, con respuestas tipo Likert que van desde nunca (1) hasta siempre (5). Asimismo, la prueba está dividida en cinco dimensiones y cada dimensión con 6 ítems, las dimensiones son: académico - laboral, social, emocional, familiar y físico. Según la validación hecha por Alfaro (2009), la confiabilidad del instrumento es altamente significativa con un valor estadístico - Alfa de Cronbach 0.858 y a través de la técnica de correlación se demostró su validez. Para el presente estudio se realizó el análisis de fiabilidad de un Alpha de Cronbach $(0,847)$ y la validez de sub test-test.

Por otro lado, para la evaluación de la conducta antisocial se tomó la prueba elaborada por Nicolás Seisdedos (2004), denominada Cuestionario (A -D) Conducta Antisociales - Delictivas. El cuestionario se aplica de manera individual o colectiva a niños y adolescentes de 11 a 19 años con una duración de 15 minutos aproximadamente. Además, la prueba está dividida en dos dimensiones claramente diferenciadas: dimensión antisocial que consta de 20 ítems y la dimensión de conducta delictiva o delincuente con 20 ítems. Para lainvestigación se utilizó solo las preguntas de la primera dimensión puesto que solo se busca correlacionar la variable de conducta antisocial. Para la presente investigación se toma la prueba adaptada por (Jullon, K., 2014), También se realizó el análisis de fiabilidad en el que se evidencia un coeficiente 0,696 y la validez de ítem - test.

\subsection{Análisis estadístico}

Para el análisis de los datos de utilizó el programa de estadístico SPSS 22, la 
prueba estadística Kolmogorov -Smimov (K-S) y finalmente se empleó el coeficiente Rho de Sperman para hallar la correlación entre las variables.

\section{Resultados.}

En la tabla 2 se observa los resultados de la prueba de bondad de ajuste de Kolmogorov - Smimov (K-S) se encontró que los datos en su mayoría no presentan una distribución normal dado que el coeficiente obtenido (K-S) no es significativa $(p<0,05)$. Por lo tanto, para el análisis de correlación se empleó el estadístico no paramétrico.

Tabla 2

Prueba de bondad de ajuste a la curva para las variables de estudio

\begin{tabular}{llllll}
\hline Instrumento & Variables & Media & D.E. & K-S & P \\
\hline \multirow{6}{*}{ Autoconcepto } & Autoconcepto general & 99.29 & 13.32 & 0.143 &, $000^{\text {c }}$ \\
& Académico & 19.88 & 4.116 & 0.161 &, $000^{\text {c }}$ \\
& Social & 22.31 & 3.836 & 0.094 &, $008^{\text {c }}$ \\
& Emocional & 15.3 & 3.415 & 0.073 &, $094^{\mathrm{c}}$ \\
& Familiar & 19.97 & 5.796 & 0.074 &, $092^{\mathrm{c}}$ \\
Conducta antisocial & Físico & 21.83 & 3.421 & 0.132 &, $000^{\mathrm{c}}$ \\
& & 10,15 & 3,65 & 0,144 &, $000^{\mathrm{c}}$ \\
\hline
\end{tabular}

Como se puede apreciar en la tabla 3, el coeficiente de correlación de Spearman indica que existe relación negativa y significativa entre el autoconcepto y la conducta antisocial $\left(\mathrm{r}=-, 249^{* *} \mathrm{p}<0,005\right)$ es decir a mayor conducta antisocial menor será el autoconcepto general del adolescente. De manera similar existe relación significativa entre conducta antisocial con las dimensiones académico y emocional $\left(\mathrm{r}=-, 250^{* *} \mathrm{p}<\right.$ 0,005 y r $=-, 490^{* *} \mathrm{p}<0,005$ respectivamente). Por otro lado, no existe relación entre conducta antisocial con las dimensiones social, familiar y físico. 
Tabla 3

Coeficiente de correlación entre autoconcepto y la conducta antisocial

\begin{tabular}{cll}
\hline \multirow{2}{*}{ Autoconcepto } & \multicolumn{2}{c}{ Conducta Antisocial } \\
\cline { 2 - 3 } & rho & $\mathbf{p}$ \\
\hline Autoconcepto Global &,$- 249^{* *}$ & .005 \\
Académico &,$- 250^{* *}$ & .005 \\
Social & .012 & .898 \\
Emocional &,$- 490^{* *}$ & .000 \\
Familiar & -.081 & .372 \\
Físico & -.117 & .193 \\
\hline
\end{tabular}

\section{Discusión}

Las conductas antisociales en la adolescencia se desencadenan con mayor intensidad puesto que esta etapa es considerada por diferentes autores como una etapa de cambios, y el autoconcepto cumple una función psicológicamente fundamental en el bienestar y la posterior integración social del adolescente. En este sentido los resultados de la investigación se desarrollaron de acuerdo a las hipótesis planteadas.

En cuanto a la hipótesis general, resultados de la investigación evidencian que existe relación inversa altamente significativa $\left(\mathrm{rho}=-, 249^{* *}\right.$ y $\left.\mathrm{p}<0,005\right)$ entre el autoconcepto general y la conducta antisocial, es decir que a menor autoconcepto mayor conducta antisocial. Estos resultados coinciden con la investigación desarrollada por García y Méndez (2016) con las mismas variables. Además, diversos autores señalan que los adolescentes con bajo autoconcepto demuestran conductas agresivas, de burla o abuso hacia los demás, así como desajuste emocional que provoca la fácil alteración a las demandas del entorno, presencia de dificultades escolares que no le permiten una adaptación e integración social adecuada. Además, les lleva a percibirse menos valorados por sus docentes o superiores lo que conlleva una mayor insatisfacción con la vida y sentimientos de soledad (Garaigordobil y Dura, 2006; Moreno Estévez, Murgui y Musitu, 2009).

En cuanto al análisis entre el autoconcepto académico/laboral y la conducta antisocial, los resultados muestran que existe relación inversa altamente significativa con un rho de $-0,250^{* *}$ y $\mathrm{p}<0.005$. Lo que indica que los adolescentes que se desarrollan en el ámbito académico y/o laboral, que piensan y sienten que sus superiores o profesores tienen un concepto inadecuado de ellos y del trabajo que desarrollan, hará que los adolescentes incurran en conductas antisociales tales como negarse a hacer las tareas 
encomendadas, plagiar durante los exámenes, llegar tarde, salir sin permiso, hacer ruido en clases, hurtar, mentir, contestar inadecuadamente a los docentes y superiores, entre otras conductas. García y Musitu (2001) explican que el autoconcepto académico/laboral es la percepción que el individuo tiene de la calidad del desempeño como estudiante y/o trabajador y gira entorno a dos ejes semánticos: el primero es el sentimiento que el adolescente tiene del desempeño de su rol a través de sus profesores o superiores, y el segundo se refiere a la calidad y el valor de su trabajo, al no percibir positivamente estos aspectos puede darse conductas desadaptativas que alteran el desarrollo del individuo y la sociedad. Estas alteraciones de conducta son una realidad en la sociedad actual, se desarrollan en los centros educativos y su entorno cercano, donde los adolescentes presentan baja tolerancia a la frustración, irresponsabilidad y optan por conductas antisociales como medios de escape, siendo estas reforzados por sus pares (Rutter, Giller y Hagell, 2000).

De la misma manera en cuanto al autoconcepto social y la conducta antisocial en el adolescentes no se encontró una relación significativa (rho $=0,012$ p>0, 898). El autoconcepto social está definido, como la percepción que tiene el sujeto de su desempeño en las relaciones sociales y se dividen en dos ejes, donde el primero hace referencia a la red social del sujeto y a su facilidad para mantenerla o ampliarla, y el segundo a las cualidades importantes de los sujetos en las relaciones interpersonales. En la investigación se encontró que un autoconcepto social adecuado no determina la conducta, por ende ser o no amigables, alegre, tener o no amigos, no es problema para mantener o ampliar sus relaciones sociales, puesto que se sabe que los adolescentes reciben aceptación de sus pares por las conductas antisociales que muestran como por ejemplo hacer bromas pesadas a la gente empujándolas a charcos o quitándoles la silla cuando se van a sentar, llamar a la puerta de alguien y salir corriendo, pelearse con otros y decir groserías o palabras fuertes. Según Alcázar (2007) los adolescentes antisociales tienen pocos amigos y los que poseen, son también adolescentes con conductas antisociales y delincuentes. Por otro lado Garaigordobil, Cruz, y Pérez, (2003); Rugby y Slee, (1993) refieren que las conductas prosociales de ayuda y de respeto favorecen a que el adolescente posea un autoconcepto social saludable y este podría modular el impacto negativo de los problemas psicológicos de conducta. De la misma forma en la investigación realizada por Alfaro (2009) no se encontró relación 
en esta dimensión por que no está definida la identidad social en los adolescentes.

Por otro lado, los resultados de la investigación muestran que no existe relación significativa entre la dimensión familiar y la conducta antisocial en los adolescentes $($ rho $=-.081$ p > 0,372). Para García y Musitu (2001) la dimensión autoconcepto familiar está determinada por la confianza y el afecto en relación a los padres, asimismo hace referencia la felicidad, apoyo y la aceptación por los otros miembros de la familia. En este sentido, se infiere que los factores predisponentes para estas conductas pueden ser el pertenecer a familias disfuncionales, la ausencia de una figura paterna y adolescentes en estado de abandono. Estos factores de riesgo imposibilitan al adolescente a tener definido un autoconcepto saludable, por ende el sentirse o no queridos por los padres, el sentir o no la ayuda de la familia cuando tienen problemas y el sentirse o no feliz en el hogar, además de ser o no criticados en casa, que la familia del adolescente este o no decepcionado de él y que los padres le brinden o no confianza no determina su conducta antisocial. Los resultados de la presente investigación hacen referencia a que la supervisión y el control de parte de los padres no es factor significativo para la prevención de conductas antisociales y delictivas, especialmente en el caso de los adolescentes varones. Esto difiere con lo mencionado por Angenent y De Mann, (1996) quienes afirman que la supervisión y el monitoreo de los padres parece ser un factor significativo para la prevención de delitos. Por otro lado Sakloíske y Zeidener (1987) encontró que los adolescentes que incurren a conductas antisociales y delictivas tienen familiares que están implicados en delitos, por ejemplo el arresto de un familiar, particularmente del padre o de alguno de la misma generación, incrementa la probabilidad de que algún miembro de la familia de la siguiente generación (hijos, sobrinos, nietos) sea delincuente, es por esto que el autoconcepto familiar no determina la conducta antisocial.

Asimismo, respecto al quinto objetivo de la investigación, no se encontró relación entre el autoconcepto físico y la conducta antisocial en los adolescentes (rho= -.117 p> 0,193 ) esto quiere decir que el autoconcepto físico no determinara que el adolescente incurra en conductas antisociales. Esto hace referencia a que los adolescentes que presentan conducta antisocial como entrar en sitios prohibidos, romper o tirar cosas de otra persona, hacer pintas en lugares públicos y molestar y engañar a personas extrañas, no afecta su manera de percibirse físicamente. Asimismo, se infiere de la población que 
los factores socioculturales a los que pertenecen los adolescentes hace que el autoconcepto físico no tenga una definición adecuada, es decir que los jóvenes entienden por ser atractivo, elegante y un cuidado físico de acuerdo a su contexto cultural. Por otro lado, Eysenck (1996) postula respecto a la dimensión psicoticismo de la personalidad que se ve claramente relacionada con las conductas antisociales y las características de este este tipo de personalidad están dadas por la agresividad, frialdad, baja sociabilidad y el egocentrismo.

Por último, se encontró una relación negativa, altamente significativa entre el autoconcepto emocional y la conducta antisocial en adolescentes $\left(\mathrm{rho}=-, 490^{* *}\right.$ y $\mathrm{p}<$ $0,00)$ esto quiere decir que cuando existe bajo autoconcepto emocional y se evidencia mayor presencia de conducta antisocial, es decir, al adolescente se le hace difícil controlar las situaciones y emociones fuertes, respondiendo inadecuadamente y con nerviosismo a los diferentes momentos de su vida. En este sentido, Gonzales (2011) explica que los factores cognitivos internos están relacionados con la conducta antisocial y el autoconcepto emocional, por otro lado Quay (1987) indica que la mayor parte de los casos de delitos violentos y crimen se asocian más con factores internos y con mayor perturbación psicológica. Es importante mencionar que las características cognitivas dan como resultados dos caminos, por un lado la aparición de conductas antisociales si éstas características son inadecuadas y por otro el desarrollo equilibrado del sujeto y buena salud mental si son adecuadas. De la misma forma en la investigación desarrollada por Vázquez, Mohamed-Mohand y Vilariño (2008) se encontró relación significativa en la dimensión de autoconcepto emocional con la conducta antisocial en adolescentes recluidos en un centro penitenciario a diferencia de los adolescentes normativos.

\section{Referencias}

Alcázar, M. (2007). Patrones de conducta y personalidad antisocial en adolescentes. Estudio transcultural: el salvador, México, y España (Tesis de Doctorado). Universidad autónoma de Madrid, Madrid. Recuperado de https://repositorio.uam.es/bitstream/handle/10486/1702/6668_alcazar_corcoles.pdf?se quence $=1$

Alfaro, R.M. (2009). Autoconcepto y conducta disciplinaria en los alumnos del nivel secundario de la Institución Educativa Adventista Miraflores (Tesis de Maestría). Universidad Peruana Unión, Lima, Perú.

Angenent, H. y De Mann, A. (1996). Antecedentes y factores de la delincuencia 
juvenil. Nueva York: Peter Lang

Carrión, E. (2015). Clima social escolar en estudiantes del distrito de Frorencia de Mora (Tesis de Lincenciatura). Universidad Cesar Vallejo, Trujillo, Perú. Recuperado de https://es.scribd.com/doc/299610751/Conductas-Antisociales-y-Clima-Social-Escolar- enestudiantes-del-distrito-de-Florencia-de-Mora

Esnaola, I., Goñi, A. y Madariaga, J.M. (2008). El autoconcepto: perspectivas de investigación. Revista de Psicodidáctica, 13(1), 179-194. Recuperado de https://www.researchgate.net/publication/286049957

Fuentes, M., Garcia, J., Gracia, E. y Lila, M. (2011). Autoconcepto y ajuste psicosocial en la adolescencia.Revista Psicothema, 23(1), 7-12. Recuperado de http://www.psicothema.com/pdf/3842.pdf

Ministerio del interio. (2013). Plan Nacional de Seguridad Ciudadana 2013-2018. Perú:

Garaigordobil, M., Cruz, S. y Pérez, J. (2003). Análisis correlacional y predictivo del autoconcepto con otros factores conductuales, cognitivos y emocionales de la personalidad durante la adolescencia. Estudios de Psicología, 24(1), 113-134. Recuperado de http://www.sc.ehu.es/ptwpefej/publicaciones/Estudios24(1).pdf

García y Mendez (2016). Autoconcepto y conducta antisoacial en alumnos de secundaria. Investigación en ámbito escolar: Edita: ASUNIVEP, 2, 35-40. Recuperado de http://www.todostuslibros.com/libros/investigacion-en-el-ambito-escolar-unacercamiento-multidimensional-a-las-variables-psicologicas-y-educativas-volumenii_978-84-617-5570-7

García, F. y Musitu, G. (2001). Autoconcepto forma 5. Madrid: TEA Ediciones, S.A.

Garaigordobil, M. y Durá, A. (2006). Relaciones del autoconcepto y la autoestima con la sociabilidad, estabilidad emocional y responsabilidad en adolescentes de 14 a 17 años. Análisis y Modificación de Conducta, 32, 37-64. Recuperado de http://www.sc.ehu.es/ptwgalam/art_completo/autoconcepto1417.pdf

Gonzales, M. (2011). Psicología clínica de la infancia y de la adolescencia. España: Editorial Piramide.

Herrera, D. y Morales, H. (2005). Comportamiento antisocial durante la adolescencia: teoría, investigación y programas de prevención. Revista de Psicología de La PUCP, 23(2), 201-247. Recuperado de revistas.pucp.edu.pe/index.php/psicologia/article/download/2317/2266

Krug, E. G., Dahlberg, L. L., Mercy, J. a, Zwi, a B. y Lozano, R. (2003). Informe mundial sobre la violencia y la salud. Revista Do Instituto de Medicina Tropical de São Paulo, 45: 130-130. Recuperado de www.scielo.br/pdf/rimtsp/v45n3/16172.pdf

Kazdin, A. y Buela-Casal, G. (2002). Conducta antisocial. Esapaña: Piramide S.A.

Moreno, D., Estévez, E., Murgui, S. y Musitu, G. (2009). Reputación social y violencia relacional en adolescentes: el rol de la soledad, la autoestima y la satisfacción vital. Psicothema, 21, 537-542. Recuperado de www.psicothema.com/pdf/3667.pdf

Quay, H. (1987). Patrones de comportamiento delincuente: Manual de delincuencia juvenil. Nueva York: John Wiley \& Sons.

Portocarrero, Y. (2014). Socialización parental y conductas antisociales - delictivas en alumnos de tercero, cuarto y quinto grados. Revista Investig. Altoandin, 6(1), 136-176. Recuperado de huajsapata.unap.edu.pe/ria/index.php/ria/article/view/100/91 
Reckless, W. C. (1961). A new theory of delinquency and crime. Federal Probation, 25, 4246. Recuperado de heinonline.org/HOL/LandingPage?handle=hein.journals/fedpro25\&div=75\&id=\&page=

Rigby, K. y Slee, P. (1993). Dimensiones de la relación interpersonal entre los niños australianos e implicaciones para el bienestar psicológico. Revista de Psicología Social, 133 (1), 33-42. Recuperado de https://www.ncbi.nlm.nih.gov/pubmed/8464217

Rigby, K. y Slee, P. (1993). Dimensiones de la relación interpersonal entre los niños australianos e implicaciones para el bienestar psicológico. Revista de Psicología Social, 133 (1), 33-42. Recuperado de https://www.ncbi.nlm.nih.gov/pubmed/8464217

Rutter, M., Giller, H., y Hagell, A. (2000). La conducta antisocial de los jóvenes. Madrid:Cambridge Universtity Press.

Sakloíske, D y Zeidener, M. (1987). International Handbook of Personality and intelligence. New York: Boston, MA: Springer US. Recuperado de https://books.google.com.pe/books?id=FFasBgAAQBAJ\&printsec=frontcover\&dq=Inte rnational+Handbook+of+Personality+and+Intelligence\&hl=es\&sa=X\&ved=0ahUKEwj d8pX4iPDRAhXSPpAKHWgfCcYQ6AEIHTAA\#v=onepage\&q=International\%20Han dbook $\% 20$ of $\% 20$ Personality $\% 20$ and $\% 20$ Intelligence $\& \mathrm{f}=$ false

Seisdedos, N. (2004). Cuestionario A-D, Conducta antisocial-delictiva. Madrid: TEA Ediciones.

Vázquez, M; Mohamed-Mohand, L y Vilariño, M. (2008). Infractores vs . Menores normativos. Revista Galega de Cooperacion Cientifica Iberoamericana, 16, 25-30. Recuperado de https://www.researchgate.net/publication/235954016_Autoconcepto_y_comportamiento _antisocial_menores_infractores_menores_normativos 